\title{
O Nutrition Impact Symptoms (NIS) Pode Ser Utilizado Como um Instrumento de Triagem Nutricional em Pacientes com Doença Renal Crônica na População Brasileira?
}

\section{Can Nutrition Impact Symptoms (NIS) Be Used as a Nutritional Screening Tool in Patients with Chronic Kidney Disease in the Brazilian Population?}

\section{RESUMO}

Objetivo: Adaptar e validar o uso da ferramenta Nutritional Impact Symptoms (NIS) como instrumento de triagem nutricional em indivíduos com DRC na população brasileira. Metodologia: Estudo do tipo transversal desenvolvido em um Hospital Universitário, baseado nos procedimentos de validação sugeridos por Beaton et al. (2000), American Educational Research Association, American Psychological Association e National Council on Measurement in Education (2014) e revisados por Muñiz, Elosua e Hambleton (2013). Resultados: Para a validação do NIS, utilizando-se a Avaliação Subjetiva Global (ASG) como padrão, foram realizadas as análises sequenciais, obtendo-se um coeficiente da correlação de Spearman classificado como moderado $(\rho=0,51 ; p<0,001)$; um erro típico da medida absoluto de 0,46 e relativo de $30 \%$; coeficiente de variação de $15 \%$ e ausência de diferenças entre avaliações médias $(p=0,497)$. Embora a classificação entre o NIS e a ASG original tenham apresentado uma moderada correlação, sem diferenças entre as médias e um erro sistemático baixo, a concordância entre as variáveis apresentou uma amplitude alta no erro aleatório. Conclusão: ANIS foi adaptada, mas não validada para o público com doença renal em âmbito hospitalar, necessiatndo de uma avaliação com um maior número de pacientes para poder ser utilizada como parte de protocolos de avaliação nutricional com objetivo de rastreio da presença de desnutrição e posterior avaliação aprofundada da mesma.

\section{DESCRITORES}

Insuficiência Renal Crônica. Desnutrição. Avaliação Nutricional. Diálise.

\begin{abstract}
Introduction: From a nutritional point of view, malnutrition is one of the main factors that affects the prognosis of chronic kidney patients, being associated with increased morbidity and mortality in this population. Objective: To verify whether the NIS can be used as a nutritional screening tool in individuals with CKD in the Brazilian population. Methodology: Cross-sectional study developed at a University Hospital, based on the validation procedures suggested by Beaton et al. (2000), American Educational Research Association, American Psychological Association and National Council on Measurement in Education (2014) and reviewed by Muñiz, Elosua and Hambleton (2013). Results: For the validation of NIS using ASG as a standard, sequential analyses were performed, and a Spearman correlation coefficient classified as moderate was obtained $(\rho=0.51$; $p<0.001$ ); a typical error of the absolute measure of 0.46 and a relative error of $30 \%$; coefficient of variation of $15 \%$ and absence of differences between average assessments $(p=0.497)$. Although the classification between the NIS and the original SGA has a moderate correlation, with no differences between the means and a low systematic error, the agreement between the variables has a high amplitude in the random error. Conclusion: The NIS was adapted, but not validated for the public with kidney disease in hospitals, needing an evaluation with a larger number of patients to be able to be used as part of nutritional assessment protocols with the objective of screening for the presence of malnutrition and subsequent in-depth evaluation.
\end{abstract}

DESCRIPTORS

Chronic Renal Failure. Malnutrition. Nutritional Assessment. Dialysis.

${ }^{1}$ Nutricionista. Especialista em Urgência e Emergência pela Universidade Federal de Goiás. Especialista em Hematologia e Hemoterapia pela Universidade Federal de Goiás. Hospital das Clínicas da Universidade Federal de Goiás /EBSERH. Goiânia, Goiás, Brasil.

${ }^{2}$ Nutricionista. Mestre em Nutrição e Saúde pela Universidade Federal de Goiás. Hospital das Clínicas da Universidade Federal de Goiás/ EBSERH. Goiânia, Goiás, Brasil.

${ }^{3}$ Pós-graduada em Nutrição Clínica e Especialista em Hematologia e Hemoterapia. Hospital das Clínicas da Universidade Federal de Goiás/EBSERH. Goiânia, Goiás, Brasil.

${ }^{4}$ Nutricionista. Doutora em Ciências da Saúde pela Faculdade de Medicina da Universidade Federal de Goiás. Pontifícia Universidade

Católica de Goiás. Goiânia, Goiás, Brasil. 
$\mathrm{O}$ número de pacientes com doença renal crônica (DRC) vem aumentando de maneira expressiva nas duas últimas décadas. Segundo o Global Kidney Disease 3, a prevalência estimada de indivíduos com diferentes graus de disfunção renal (estágios de 1 a 5) em diversos países varia de 8 a 16\%. Tal fato pode estar associado ao envelhecimento populacional e à elevada prevalência de doenças de base, como o diabetes mellitus e a hipertensão arterial sistêmica. Consequentemente, a proporção de pacientes em diálise com múltiplas comorbidades também aumentou ${ }^{1,2}$.

Além das causas patológicas da DRC, como o diabetes mellitus e a hipertensão arterial outros fatores podem ser associados ao surgimento desta patologia. A influência natural do envelhecimento pode ser destacada neste processo, pois afeta todos os componentes do rim, levando assim, a alterações na morfologia renal e a diminuição de sua função, podendo ser agravadas pela presença de comorbidades ${ }^{3}$.

Do ponto de vista nutricional, a desnutrição é um dos principais fatores que afetam o prognóstico do paciente renal crônico, sendo associada ao aumento da morbimortalidade nessa população ${ }^{4}$. Em pacientes com DRC, existe uma relação multifatorial entre a inflamação e a desnutrição. Alguns fatores como distúrbios metabólicos, processo dialítico e anorexia têm envolvimento na desnutrição, induzindo à perda proteica corporal e a liberação de citocinas pró-inflamatórias que interferem neste processo ${ }^{5}$.

Com isso, o quadro de desnutrição é agravado por restrições alimentares, as quais os indivíduos em tratamento dialítico são sub- metidos, como perda de aminoácidos no dialisato, intercorrências infecciosas, distúrbios gastrointestinais e administração de certas medicações que favorecem anormalidades no perfil nutricional dos pacientes ${ }^{6}$.

A prevalência de desnutrição em pacientes em hemodiálise (HD) varia de 10 a $60 \%^{7}$. A variação na prevalência de desnutrição nesta população pode ser atribuída aos diferentes métodos utilizados para a avaliação e aos múltiplos fatores que contribuem para o seu desenvolvimento ${ }^{8}$.

Diante da alta prevalência de desnutrição em pacientes com DRC fica evidente a importância da realização da triagem e a avaliação nutricional com o objetivo de identificar as causas de risco e/ou piora do estado nutricional. Com base nesta avaliação, busca-se traçar metas nutricionais para prevenir e/ou tratar a desnutrição. Entretanto, não existe um marcador isolado que seja capaz de avaliar o estado nutricional de pacientes com DRC, devido as diversas anormalidades inerentes a própria enfermidade ${ }^{9}$.

Os métodos objetivos de avaliação do estado nutricional, mais empregados na prática clínica em pacientes com DRC, incluem a antropometria, a bioimpedância, a força de preensão manual, os dados de ingestão alimentar e os exames laboratoriais. Entre os métodos compostos de avaliação do estado nutricional, destacam-se a avaliação subjetiva global (ASG) e o índice de desnutrição e inflamação (malnutrition inflammation score - MIS) constituindo os instrumentos subjetivos mais utilizados nesse grupo de pacientes ${ }^{10}$.

Outro instrumento de avaliação do estado nutricional consiste na avaliação subjetiva global produzida pelo paciente (ASG-PPP), 
desenvolvida prioritariamente para pacientes oncológicos. A partir dessa ferramenta, desenvolveu-se o Nutrition Impact Symptoms (NIS) que faz parte da ASG-PPP, sendo uma ferramenta validada de avaliação nutricional, desenvolvida inicialmente para uso em oncologia e, posteriormente, validado no Reino Unido para pacientes em HD. Evidências recentes, apoiam a eficácia do escore NIS como ferramenta de triagem nutricional, válida e confiável para HD e em pacientes ambulatoriais nefropatas, não sendo validado no Brasil, podendo ser utilizado como um instrumento de mais fácil aplicação e com potencial de identificar mais sensivelmente o risco nutricional nesta população, quando comparada com outras ferramentas ${ }^{11,12}$.

A partir do exposto, a fim de avaliar corretamente o estado nutricional do paciente com DRC, o NIS torna-se uma importante ferramenta de triagem nutricional, o que contribui para a instituição de planos terapêuticos mais eficazes. Assim, o objetivo do presente trabalho consiste em adaptar e validar o uso da ferramenta Nutritional Impact Symptoms (NIS) como instrumento de triagem nutricional em indivíduos com DRC na população brasileira.

\section{METODOLOGIA}

Trata-se de um estudo do tipo transversal desenvolvido em um Hospital Universitário, de adaptação e validação de um instrumento de triagem nutricional baseado nos procedimentos de validação sugeridos por Beaton et al. ${ }^{13}$, American Educational Research Association, American Psychological Association e National Council on Measurement in Education ${ }^{14}$ e revisados por Muñiz, Elosua e Ham- bleton $^{15}$, conforme quadro-resumo (Tabela 1 ).

A amostra do estudo foi selecionada por conveniência e composta por pacientes hospitalizados com diagnóstico de DRC em tratamento no Hospital Universitário, admitidos nas clínicas: médica, cirúrgica, tropical e pronto-socorro. Também foram recrutados pacientes no setor de hemodiálise. Como critérios de inclusão foram considerados indivíduos com idades entre 20 e 80 anos e com diagnóstico confirmado de DRC e foram excluídos da pesquisa pacientes em cuidados intensivos, gestantes, pacientes com membros amputados, com neoplasias e síndrome da imunodeficiência adquirida (HIV).

A coleta de dados foi realizada por nutricionistas previamente treinados, com experiência na área de nutrição clínica hospitalar, no período de março a agosto de 2019, utilizando o instrumento Nutrition Impact Symptoms (NIS), conforme procedimentos metodológicos estabelecidos na Tabela 1 deste trabalho. O NIS é dividido em 3 classificações de acordo com o resultado final de sua aplicação, sendo o risco nutricional classificado em: alto, moderado e baixo.

Após a aplicação do NIS, foi aplicada a ASG para posterior comparação com o instrumento a ser validado. De acordo com o resultado final da aplicação da ASG, o estado nutricional foi classificado em: (A) bem nutrido; (B) desnutrido leve ou moderado ou (C) desnutrido grave. Foram coletados também dados antropométricos (peso, altura, circunferência do braço e circunferência da panturrilha) e bioquímicos (proteína C-reativa e albumina). Os exames bioquímicos foram coletados do prontuário e foram considerados no estudo, os resultados mais recentes. 
Tabela1. Quadro-resumo dos procedimentos para validação de instrumentos

\begin{tabular}{c|l}
\hline Etapa & \multicolumn{1}{c}{ Procedimento } \\
\hline \multicolumn{1}{c|}{$\begin{array}{c}\text { Confiabilidade e validação } \\
\text { Evidência de } \\
\text { confiabilidade } \\
\text { baseada na } \\
\text { consistência } \\
\text { interna. }\end{array}$} & $\begin{array}{l}\text { Avaliar a confiabilidade de um teste diz respeito a verificar se o } \\
\text { mesmo pode ser usado por diferentes entrevistadores, em diferentes } \\
\text { momentos, visando um mesmo desfecho. }\end{array}$ \\
$\begin{array}{c}\text { Aconsistência interna é uma medida de confiabilidade que estabelece } \\
\text { correlação entre os itens dos testes, de forma que, cada item é visto } \\
\text { como medida única, avaliando aspectos diferentes do constructo. }\end{array}$ \\
$\begin{array}{l}\text { Neste estudo, será verificado por meio do indicador alfa de Cronbach } \\
\text { o grau de relação entre os itens dos testes e os desfechos a partir da } \\
\text { aplicação única de cada teste em uma amostra da população-alvo. }\end{array}$ \\
\hline $\begin{array}{c}\text { Evidência de } \\
\text { validade basea- } \\
\text { da na validade } \\
\text { de constructo } \\
\text { convergente. }\end{array}$ & $\begin{array}{l}\text { A avaliação de validade de um teste visa demonstrar a eficácia do } \\
\text { mesmo em medir sua proposição. }\end{array}$ \\
$\begin{array}{l}\text { A validade de constructo é utilizada quando o desfecho não é fa- } \\
\text { cilmente observável e detectável, utilizando-se de itens hipotéticos } \\
\text { baseados em teoria para prever o desfecho. Na validade de cons- } \\
\text { tructo convergente, avalia-se o quanto os instrumentos estudados } \\
\text { se correlacionam com outras variáveis e medidas relacionadas ao } \\
\text { mesmo desfecho. } \\
\text { Para verificar a validade do instrumento, serão comparados os va- } \\
\text { lores dos escores gerados pelo NIS com os escores gerados pela } \\
\text { ASG previamente validada para a população de estudo. } \\
\text { Os testes utilizados neste estudo foram elaborados com base em } \\
\text { teoria, buscando identificar os desfechos previstos, justificando assim } \\
\text { a utilização desta medida neste estudo. }\end{array}$ \\
\hline
\end{tabular}

As medidas antropométricas foram realizadas antes da aplicação do instrumento NIS. Foram aferidos o peso corporal e a altura dos indivíduos de acordo com os procedimentos descritos por Heyward e Stolarczyk ${ }^{16}$. Em seguida, foi calculado o Índice de Massa Corporal (IMC), conforme fórmula a seguir: $\left[\mathrm{IMC}=\right.$ peso $(\mathrm{kg}) /$ estatura $\left.(\mathrm{m})^{2}\right] . \mathrm{O} \mathrm{IMC}$ foi classificado para adultos, seguindo o padrão da Organização Mundial de Saúde ${ }^{17}$ e para idosos conforme Lipschitz ${ }^{18}$.

Os dados foram digitados em planilha no programa Excel $囚$ e apresentados em média \pm desvio padrão. Foi realizada análise es- tatística descritiva com obtenção de frequências, médias e desvio padrão da média. Para avaliar a confiabilidade mediante aplicação única do questionário, foi utilizado o indicador alfa de Cronbach ${ }^{19}$, seguindo recomendações de Bland e Altman ${ }^{20}$, com valores desejáveis mínimos de 0,9.

Para avaliar a correlação e a concordância dos itens foram utilizados os coeficientes de Bland-Altman e Kappa, por meio dos softwares MedCalc $₫$, Korea, Seoul. O teste de Kappa com peso quadrático foi utilizado para avaliar a concordância entre as duas classificações utilizando as variáveis 
numéricas dos escores de MRSA de 7 e 5 itens, onde $K \leq 0,20$ : concordância fraca, $K$ $\geq 0,21$ e $\leq 0,40$ : razoável, $K \geq 0,41$ e $\leq 0,6$ : moderada, $K \geq 0,61$ e $\leq 0,8$ : forte e $K \geq 0,81$ e $\leq 1,0$ : muito forte.

Foram ainda aplicados os testes de Shapiro-Wilk para verificação da normalidade; ANOVA one-way, para comparação de médias com distribuição normal; Kruskall-Wallis para comparação de médias com distribuição não-normal; e o teste Exato de Fisher para checar a associação entre distribuição de variáveis categóricas. Essas análises foram conduzidas no software STATA e foram considerados significantes os valores de $p<0,05$.

Os participantes foram convidados a participar da pesquisa e foram devidamente informados a respeito do protocolo do estudo por meio do um Termo de Consentimento Livre e Esclarecido (TCLE). Todos os procedimentos adotados pela pesquisa estão em conformidade com a Resolução n 466 , de 12 de dezembro de 2012, do Conselho Nacional de Saúde do Ministério da Saúde e Resolução $n^{\circ} 510,07$ de abril de $2016^{21,22}$.

\section{RESULTADOS}

Foram analisados 45 pacientes com DRC no período de março a agosto de 2019, com média de idade de 59,11 $\pm 15,53$ anos, dos quais $51,11 \%$ eram do sexo feminino. A maioria dos pacientes $(51,11 \%)$ apresentava estágio $V$ da DRC e não realizava HD. O tempo médio de diagnóstico foi de $45,27 \pm$ 56,71 meses e de tratamento de 40,20 $\pm 32,65$ meses.

A média de peso dos avaliados foi de
$70,26 \pm 16,93 \mathrm{~kg}$, de IMC de 26,20 5 ,89 kg/ $\mathrm{m}^{2}$, dos quais apenas $8,89 \%$ apresentavam magreza e 6,67\% obesidade. As médias de circunferência de braço (CB) e circunferência de panturrilha $(\mathrm{CP})$ foram respectivamente, $28,24 \pm 4,90 \mathrm{~cm} \mathrm{e} 32,95 \pm 4,46 \mathrm{~cm}$ (Tabela 2).

Acerca das dosagens bioquímicas, verificou-se uma média de $3,40 \pm 0,70 \mathrm{mg} / \mathrm{dL}$ de albumina sérica e 4,75 $\pm 9,09 \mathrm{mg} / \mathrm{dL}$ de PCR. Quanto à classificação de ASG a maior frequência $(51,11 \%$ ) foi de $A$ (nutrido) (Tabela 2 ).

Verificam-se diferenças estatisticamente significativas entre as médias de $\mathrm{CB}$ e $C P$, sendo que essas foram maiores nos indivíduos com baixo risco avaliado pelo NIS em comparação com os avaliados com risco moderado ou alto. O risco alto teve média de $C B$ e $C P$ maior que o risco moderado, no entanto, a média ainda foi menor que no risco baixo. Foi verificada ainda associação entre a classificação da ASG e NIS $(p=0,002)$ de modo que ocorreu maior frequência de pacientes com risco baixo avaliado pela NIS e classificado como A (nutrido pela ASG) 75 $\%)$ e menor frequência de indivíduos com risco moderado pelo NIS classificado como $B$ (suspeita de desnutrição) pela ASG.

A correlação entre as medidas de dosagens bioquímicas, antropometria e a idade dos pacientes está demonstrada na Tabela 3. De acordo com os resultados obtidos, observou-se uma correlação inversa fraca do NIS com CB $(\rho=-0,36, p=0,016)$ e CP $(\rho=-0,35, p=0,021)$ dos pacientes avaliados; e uma correlação direta fraca do NIS com a Idade $(\rho=0,35, p=0,046)$ e moderada com as concentrações séricas de PCR $(\rho=0,45$, $p=0,004)$. 
Tabela 2. Características sociais, clínicas e antropométricas dos pacientes

\begin{tabular}{|c|c|c|c|c|c|}
\hline Variáveis & $\begin{array}{c}\text { Amostra total } \\
n=45\end{array}$ & $\begin{array}{c}\text { NIS } \\
\text { Risco Baixo } \\
24(53,33)\end{array}$ & $\begin{array}{c}\text { NIS } \\
\text { Risco Moderado } \\
15(33,33)\end{array}$ & $\begin{array}{c}\text { NIS } \\
\text { Risco Alto } \\
6(13,34)\end{array}$ & $p$-valor \\
\hline Idade & $59,11 \pm 15,53$ & $55,92 \pm 15,54$ & $62,27 \pm 15,26$ & $64,00 \pm 15,82$ & $0,335^{\top}$ \\
\hline Sexo & & & & & $0,247^{*}$ \\
\hline Feminino & $23(51,11)$ & $12(50,00)$ & $6(4000)$ & $5(83,33)$ & \\
\hline Masculino & $22(48,89)$ & $12(50,00)$ & $9(60,00)$ & $1(16,67)$ & \\
\hline Grau de DRC & & & & & $0,912^{*}$ \\
\hline 1 & - & - & - & - & \\
\hline II & - & - & - & - & \\
\hline III & $9(20,00)$ & $4(16,67)$ & $4(26,67)$ & $1(16,67)$ & \\
\hline IV & $13(28,89)$ & $8(33,33)$ & $3(20,00)$ & $2(33,33)$ & \\
\hline $\mathrm{V}$ & $23(51,11)$ & $12(50,00)$ & $8(53,33)$ & $3(50,00)$ & \\
\hline Hemodiálise & & & & & $1,000^{*}$ \\
\hline Sim & $20(44,44)$ & $10(41,67)$ & $7(46,67)$ & $3(50,00)$ & \\
\hline Não & $25(55,56)$ & $14(58,33)$ & $8(53,33)$ & $3(50,00)$ & \\
\hline $\begin{array}{l}\text { Tempo de diagnóstico } \\
\text { (meses) }\end{array}$ & $45,27 \pm 56,71$ & $39,04 \pm 42,71$ & $51,93 \pm 67,69$ & $53,50 \pm 81,74$ & $0,786^{*}$ \\
\hline $\begin{array}{l}\text { Tempo de tratamento } \\
\text { (meses) }\end{array}$ & $40,20 \pm 32,65$ & $37,54 \pm 43,45$ & $50,73 \pm 67,92$ & $24,50 \pm 32,65$ & $0,518^{*}$ \\
\hline \multicolumn{6}{|l|}{ Antropometria } \\
\hline Peso & $70,26 \pm 16,93$ & $74,81 \pm 19,25$ & $64,11 \pm 13,24$ & $67,47 \pm 10,14$ & $0,178^{*}$ \\
\hline IMC & $26,20 \pm 5,89$ & $27,75 \pm 6,82$ & $24,00 \pm 4,41$ & $25,47 \pm 3,26$ & $0,152^{*}$ \\
\hline $\mathrm{IMC}$ & & & & & $0,590^{*}$ \\
\hline Magreza & $4(8,89)$ & $1(4,17)$ & $3(20,00)$ & - & \\
\hline Eutrofia & $23(51,11)$ & $11(45,83)$ & $8(53,33)$ & $4(66,67)$ & \\
\hline Sobrepeso & $15(33,33)$ & $10(41,67)$ & $3(20,00)$ & $2(33,33)$ & \\
\hline Obesidade & $3(6,67)$ & $2(8,33)$ & $1(6,67)$ & - & \\
\hline $\mathrm{CB}(\mathrm{cm})$ & $28,24 \pm 4,90$ & $30,04 \pm 4,73 a$ & $26,19 \pm 4,86 b$ & $26,42 \pm 3,17 b$ & $0,018^{\top}$ \\
\hline $\mathrm{CP}(\mathrm{cm})$ & $32,95 \pm 4,46$ & $34,61 \pm 4,46 a$ & $30,53 \pm 3,94 b$ & $32,25 \pm 3,13 a b$ & $0,020^{\top}$ \\
\hline \multicolumn{6}{|l|}{ Dosagens Bioquímicas } \\
\hline Albumina (mg/dL) & $3,40 \pm 0,70$ & $3,41 \pm 0,67$ & $3,59 \pm 0,65$ & $2,92 \pm 0,82$ & $0,202^{\top}$ \\
\hline $\mathrm{PCR}(\mathrm{mg} / \mathrm{dL})$ & $4,75 \pm 9,09$ & $1,71 \pm 2,08$ & $6,42 \pm 8,52$ & $10,47 \pm 18,47$ & $0,061^{*}$ \\
\hline \multicolumn{6}{|l|}{ Avaliações subjetivas } \\
\hline NIS & $3,75 \pm 3,34$ & $1,12 \pm 1,15 a$ & $5,53 \pm 1,24 b$ & $9,83 \pm 0,98 \mathrm{c}$ & $<0,001^{*}$ \\
\hline ASG & & & & & $0,002^{*}$ \\
\hline$A$ & $23(51,11)$ & $18(75,00)$ & $4(26,67)$ & $1(16,67)$ & \\
\hline $\mathrm{B}$ & $20(44,44)$ & $6(25,00)$ & $10(66,67)$ & $4(66,67)$ & \\
\hline $\mathrm{C}$ & $2(4,44)$ & 0 & $1(6,67)$ & $1(16,67)$ & \\
\hline
\end{tabular}

Valores apresentados em média \pm desvio-padrão da média ou valores absolutos (valores relativos). p-valor obtido por $\mathrm{T}$ teste de ANOVA one-way; ou ж teste Exato de Fisher ou * teste de Kruskall-Wallis, todos com 5\% de nível de significância.(Letras minúsculas ladeadas de suas médias e desvios padrões referem-se à diferenciação) post hoc de Bonferroni. 
Tabela 3. Correlação entre o NIS, dosagem bioquímica, antropometria e idade em pacientes brasileiros com doença renal crônica

\begin{tabular}{l|c|c|c|c|c|c|c}
\hline & Albumina & PCR & Peso & IMC & CB & CP & Idade \\
\hline Coeficiente Rho & $-0,17$ & 0,45 & $-0,21$ & $-0,17$ & $-0,36$ & $-0,35$ & 0,30 \\
\hline p-valor & 0,347 & 0,004 & 0,159 & 0,263 & 0,016 & 0,021 & 0,046 \\
\hline
\end{tabular}

p-valor obtido por teste de correlação de Spearman, com 5\% de nível de significância.

A Tabela 4 apresenta a ocorrência de sintomas avaliados pelo NIS. A xerostomia foi o sintoma mais frequente, com $64,44 \%$ $(n=29)$ da amostra total, seguida pela perda do apetite, com $46,66 \%$ ( $n=21)$.

Para a análise de concordância entre as classificações avaliadas pelo NIS e pela ASG foi estimado o valor de Kappa, que revelou uma razoável e significante concordância entre eles (Kappa $=0,380$, concordância de $64,44 \%$, erro padrão $=0,118, p<0,001)$. A confiabilidade entre a classificação do NIS e da ASG, pelo teste alfa de Cronbach, seguindo o modelo de confiabilidade de forma paralela, evidenciou confiabilidade substancial entre os questionários, com um coeficiente alfa de 0,68 e padrão alfa de 0,51.

Para a validação do NIS, utilizando a ASG como padrão, foram realizadas as análises sequenciais e obteve-se um coeficiente da correlação de Spearman classificado como moderado $(\rho=0,51 ; p<0,001)$; um erro típico da medida absoluto de 0,46 e relativo de $30 \%$; coeficiente de variação de $15 \%$ e ausência de diferenças entre avaliações médias $(p=0,497)$; e as avaliações absolutas e relativas do Bland-Altman estão apresentadas na figura a seguir (Figura 1 e 2, respectivamente).

Tabela 4. Ocorrência de sintomas identificados pelo NIS na amostra

\begin{tabular}{l|cc}
\hline \multicolumn{1}{c|}{ Sintomas } & $\mathrm{n}=45$ & $\%$ \\
\hline Xerostomia & 29 & 64,44 \\
Perda do apetite & 21 & 46,66 \\
Náuseas & 15 & 33,33 \\
Constipação & 14 & 31,11 \\
Vômitos & 10 & 22,22 \\
Plenitude gástrica & 10 & 22,22 \\
Dor & 09 & 20 \\
Alteração do paladar & 08 & 17,77 \\
Os cheiros enjoam & 07 & 15,55 \\
Diarreia & 04 & 8,88 \\
Feridas na boca & 03 & 6,66 \\
Problemas para engolir & 03 & 6,66 \\
Outros & 02 & 4,44
\end{tabular}


Figura 1. Avaliação absoluta do Bland Altman

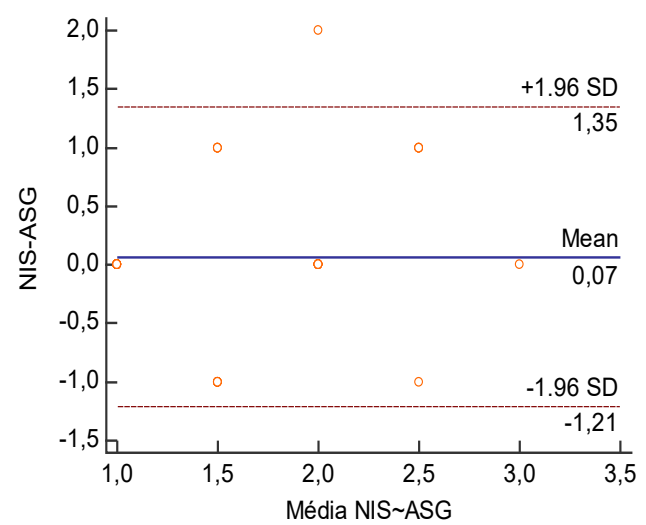

Dessa forma, embora a classificação entre o NIS e a ASG original apresentem moderada correlação, sem diferenças entre as médias e o erro sistemático baixo, a concordância entre as variáveis apresenta uma amplitude alta no erro aleatório.

\section{DISCUSSÃO}

O presente estudo verificou que a pontuação do NIS não pode ser considerada uma ferramenta válida de triagem nutricional para avaliar o risco de desnutrição em pacientes nefropatas na população brasileira, diferente de outros trabalhos em outras populações ${ }^{11,12}$. O instrumento foi adaptado, porém não validado para esta categoria de pacientes.

Os guias de condutas para pacientes com DRC como o National Kidney Foundation/ Clinical Practices Guidelines for Chronic Kidney Disease (Nutrition) (Nutrition K/DOQI), o European Best Practice Guidelines in Nutrition (EBPG) e as Diretrizes de Terapia Nutricional para pacientes em HD da Sociedade Brasileira de Nutrição Parenteral e Enteral (SBNPE)
Figura 2. Avaliação relativa do Bland Altman

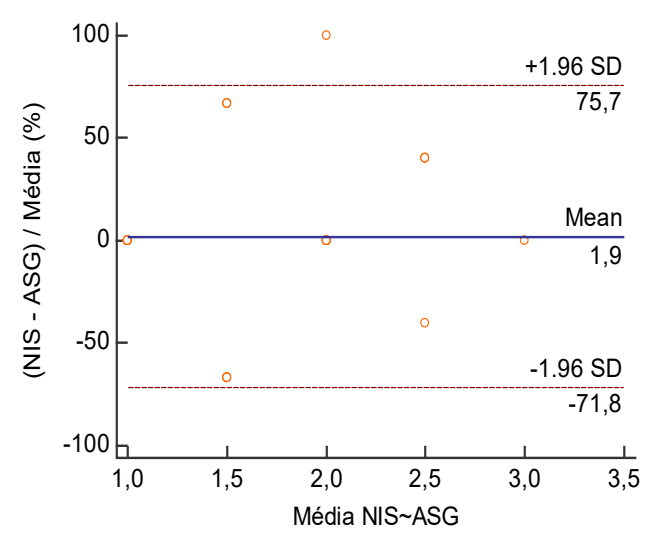

recomendam que sejam utilizados métodos objetivos e subjetivos para avaliar o estado nutricional do paciente, obtendo assim diagnóstico nutricional mais preciso ${ }^{10}$.

Com isso, ferramentas de avaliação nutricional são projetadas para auxiliar os profissionais nos serviços de saúde na detecção da desnutrição. Uma vez identificada, intervenções nutricionais precoces podem ser implementadas com a finalidade de reduzir ainda mais a deterioração e declínio no estado nutricional ${ }^{23}$.

A pontuação do NIS identifica fatores específicos relacionados à desnutrição, orientando a escolha de intervenção clínica. Alguns estudos conseguiram demonstrar a flexibilidade da pontuação do NIS como uma ferramenta de triagem em diferentes pacientes com DRC, sendo de fundamental importância, a validação dessa ferramenta de triagem dentro da população brasileira ${ }^{11,12}$.

O desenvolvimento de uma ferramenta de triagem nutricional rápida e objetiva, que pudesse ser facilmente administrada seria clinicamente valioso. Assim sendo, o uso de 
uma ferramenta com foco no monitoramento dos sintomas, como o NIS, pode ajudar a equipe multidisciplinar a identificar rapidamente pacientes em risco nutricional com DRC ${ }^{12}$.

Existem várias vantagens no uso do NIS como ferramenta de triagem nutricional em detrimento de outras ferramentas. Ele não requer medição do peso corporal, conhecimento do peso livre de edema ou perda de peso anterior. Ele também não possui parâmetros bioquímicos incluídos e, portanto, pode ser rápido e facilmente realizado à beira leito. Além disso, o instrumento pode identificar os principais fatores que impactam na ingestão de alimentos precocemente durante a hospitalização, podendo assim orientar as intervenções para melhorar o estado nutricicional ${ }^{12}$.

Em um estudo realizado no Reino Unido, utilizando o NIS como ferramenta de triagem nutricional, observou-se uma sensibilidade de $89 \%$ e uma especificidade de $65 \%$ do instrumento quando comparado com a ASG, sendo recomendado o seu uso pela fácil aplicação e a alta sensibilidade em identificar a desnutrição em pacientes com DRC ${ }^{11}$.

A ASG, que foi utilizada neste estudo para a comparação com o NIS, é um instrumento que engloba tanto aspectos subjetivos quanto objetivos da história clínica e física do paciente, no qual pode ser aplicada em pacientes com DRC. A ferramenta possui muitos pontos fortes na utilização clínica: possui baixo custo, de rápida aplicação e pode ser usada efetivamente por qualquer profissional da saúde bem treinado, sendo uma ferramenta reproduzível, válida e confiável ${ }^{24}$.

Porém, a aplicação da ASG apresenta desvantagens como a incapacidade do méto- do de demonstrar claramente a diminuição de risco nos pacientes desnutridos graves, apenas em uso de terapia nutricional. Outra limitação é sua utilização para monitorar a evolução dos pacientes, uma vez que a ASG é baseada exclusivamente em critérios qualitativos, onde pequenas alterações do estado nutricional não são detectadas ${ }^{5,25}$. Portanto, mediante as limitações da ASG, verificou-se a importância da aplicação de uma ferramenta de triagem nutricional específica para nefropatas.

Quando se trata de métodos compostos de avaliação do estado nutricional é importante verificar se os mesmos são capazes de diagnosticar corretamente a condição de desnutrição. Portanto, deve-se avaliar se esses métodos apresentam boa sensibilidade (verdadeiro positivo) e especificidade (falso positivo) para esse fim. Quando existe um método ouro para avaliar o estado nutricional, a sensibilidade e especificidade são avaliadas encontrando o ponto de corte ou valor com melhor capacidade de predizer o desfecho ${ }^{26}$.

A terapia da pessoa com DRC em realização de HD inclui restrições tanto na dieta quanto na ingestão hídrica, sendo as principais dificuldades sentidas pelos pacientes. A recomendação é que o paciente ingira $500 \mathrm{ml}$ de líquidos além da sua diurese em 24 horas, porém os pacientes anúricos tem maior dificuldade em administrar a sede. Como demonstrado neste estudo a xerostomia foi o sintoma mais prevalente entre os pacientes avaliados, resultante provavelmente da restrição hídrica a qual esses pacientes são submetidos ${ }^{27}$.

Além de auxiliar no diagnóstico do estado nutricional, identificando fatores de risco clinicamente controláveis, os instrumen- 
tos de triagem e a avaliação nutricional são muito importantes para o planejamento de intervenções específicas. No entanto, dada a multicausalidade da desnutrição na DRC, devem ser consideradas as relações entre seus determinantes, na implementação de estratégias de prevenção, diagnóstico e controle da $\mathrm{DRC}^{28}$.

O benefício de isolar as possíveis causas associadas ao consumo insuficiente de alimentos fornece uma indicação para um plano de manejo futuro. A inflamação tem sido associada aos sintomas de falta de apetite, náusea e comprometimento da função olfativa. O acúmulo de toxinas urêmicas entre as sessões de diálise tem sido associado à disgeusia, responsável por maior sensibilidade aos gostos amargos e salgados e menor sensibilidade a sabores doces. Portanto, não afeta apenas a motivação para a ingestão adequada, mas também a palatabilidade dos alimentos, sendo importante identificá-los. Essa investigação clínica indica que um sistema de questionamento simples sobre sintomas, fornece uma boa indicação das áreas nas quais os pacientes estão mais propensos a ter pior estado nutricional e ter resultados clínicos futuros ruins ${ }^{29,30}$.

Mesmo não apresentando significância, verificou-se neste estudo, que com o aumento da idade, ocorreu também um aumento da pontuação do NIS, sendo um dado importante relacionando o envelhecimento e o estado nutricional. Este dado demonstra que quanto maior a idade, mais sintomas o paciente com DRC irá apresentar, o que afetará diretamente sua ingestão nutricional.
A maior parte dos pacientes tive um bom estado nutricional, demonstrado pela ASG, CP $>31 \mathrm{~cm}$, valores de albumina e IMC médio. A maioria dos pacientes do estudo não fazia $\mathrm{HD}$, o que pode justificar o melhor estado nutricional desses indivíduos.

Entre as limitações do presente estudo podemos destacar o tamanho da amostra e o fato de que em cada avaliação o NIS e a ASG foram realizados pelo mesmo pesquisador em um único atendimento. Assim não foi possível cegar os avaliadores para os resultados dos componentes individuais.

Pesquisas futuras sobre o uso do NIS como ferramenta de triagem nutricional devem focar, além da validação, no efeito do treinamento e no envolvimento da equipe multiprofissional na implementação clínica e também os resultados de sua aplicação com foco no paciente, como qualidade de vida e capacidade funcional na alta.

Estudos longitudinais com maior tamanho amostral, incluindo diferentes faixas etárias, tendem a esclarecer o melhor uso do questionário para pacientes com doença renal crônica.

\section{CONCLUSÃO}

Conclui-se que o NIS foi adaptado, mas não validado para o público com doença renal em âmbito hospitalar, precisando de uma avaliação com um maior número de pacientes para poder ser utilizada como parte de protocolos de avaliação nutricional com objetivo de rastreio da presença de desnutrição e posterior avaliação aprofundada da mesma. 


\section{REFERÊNCIAS}

1. Castro MCM. Tratamento conservador de paciente com doença renal crônica que renuncia à diálise. J Bras Nefrol. São Paulo. 2018; 41(1):1-8.

2. Vivekanand JHA, et al. Chronic kidney disease: global dimensions and perspectives. J. Lancet, Londres. 2013; 382(1):260-272.

3. Magalhães FG, Goulart RM, Prearo LC. Impacto de um programa de intervenção nutricional com idosos portadores de doença renal crônica. Ciênc Saúde Colet. 2018; 23(8):2555-2564.

4. Martins ECV, Pereira VF, Sales PS, Pereira PAL. Tempo de hemodiálise e o estado nutricional em pacientes com doença renal crônica. Braspen J. 2017; 32(1):54-57.

5. Barros A, Sussela AO, Felix R, Lucas LS, D'Avila DO. Pacientes em hemodiálise: estado inflamatório emassa magra corporal. Sci Med. 2014; 24(1):6-10.

6. Santos ACB, Machado MC, Pereira LR, Abreu JLP, Lyra $M B$. Associação entre qualidade de vida e estado nutricional em pacientes renais crônicos em hemodiálise. $J$ Bras Nefrol. 2013; 35(4):279-188.

7. Mutsert R, Grootendorst DC, Boeschoten EW, Brandts H, Van Manen JG, Krediet RT, ET AL.; Netherlands Cooperative Study on the Adequacy of Dialysis-2 Study Group. Subjective global assessment of nutritional status is strongly associated with mortality in chronic dialysis patients. Am J of Clin Nutr, Bethesda. 2009; 89(3):787-793.

8. Oliveira CMC, Kubrusly M, Mota RS, Silva CAB, Oliveira VN. Desnutrição na insuficiência renal crônica: qual o melhor método diagnóstico na prática clínica? J Bras Nefrol. 2010; 32(1):57-70.

9. Vegine PM, Fernandes ACP, Torres MRSG, Silva MIB Anesani CM. Avaliação de métodos para identificar desnutrição energético protéica de pacientes em hemodiálise. J Bras Nefrol. 2011; 33(1):55-61.

10. Fetter RL, Bigogno FG, Oliveira FGP, Avesani CM. Adaptação transcultural para o português de instrumentos de avaliação do estado nutricional de pacientes em diálise. J Bras Nefrol. 2014; 36(2):176-185.

11. MaClaughlin HL, Twomey J, Saunt R, BlainS, Campbell $\mathrm{KC}$, Emery $\mathrm{P}$. The nutrition impact symptoms (NIS) score detects malnutrition risk in patients admitted to nephrology wards. J Hum Nutr Diet. 2018; 1(1):1-6.

12. Campbell KL, Bauer JD, Ikehiro A. et al. Role of nutrition impact symptoms in predicting nutritional status and clinical outcome in hemodialysis patients: a potential screening tool. J Ren Nutr. 2013; 23(1):302-307.

13. Beaton DE, Bombardier C, Guillemin F, Ferraz MB. Guidelines for the Process of Cross-Cultural Adaptation of Self-Report Measures. Spine J., Lebanon, 2000; 25(24):3186-3191
14. American Educational Research Association, American Psychological Association, National Council on Measurement in Education (United States). Standards for educational and psychological testing. New York, NY: AERA, APA, NCME, 2014. 11 p.

15. Muñiz J, Elosua P, Hambleton RK. Directrices para la traducción y adaptación de los tests: segunda edición. Psicothema, Oviedo, 2013; 25(2):151-157.

16. Heyward VH, Stolarczyk LM. Avaliação da composição corporal aplicada: fundamentos da composição corporal. São Paulo: Manole, 2000.

17. World Health Organization. Physical Status: The Use and Interpretation of Antropometry. Who Technical Report Series 854. Geneva, 1995. 452 p.

18. Lipschitz DA. Screening for nutritional status in the elderly. Prim Care, Philadelphia, 1994; 21(1):55-67.

19. Cronbach LJ. Coefficient alpha and the internal structure of test. Psychometrika. 1951.

20. Bland JM \& Altman DG. Cronbach's Alpha. Br Med J., 314, 572. 1997.

21. Brasil. Conselho Nacional de Saúde. Resolução 466/12. Trata de pesquisas em seres humanos e atualiza a resolução 196. [Internet]. Diário Oficial da União. 12 dez. 2012.

22. Brasil. Conselho Nacional de Saúde. Resolução n 510 , 07 de abril de 2016. Dispõe sobre as normas aplicáveis a pesquisa em Ciências Humanas e Sociais. Brasília, DF: CNS, 2016.

23. Vero LM, Byham-Gray L, Parrott JS, Steiber Al. Use of the subjective global assessment to predict health-related quality of life in chronic kidney disease stage 5 patients on maintenance hemodialysis. J Renal Nutr. 2013; 23(1):141-147.

24. Steiber AL.; Kalantar-Zadeh K, Secker D, Mccarthy M, Sehgal A, Mccann L. Subjective Global Assessment in chronic kidney disease: a review. J Renal Nutr. 2004; 14(1):191-200.

25. Barbosa-Silva MCG, Barros AJD. Avaliação nutricional subjetiva. Parte 1 - Revisão de sua validade após duas décadas de uso. Arq Gastroenterol., São Paulo, 2002; 36(3):181 -187.

26. Bignono FG, Fetter RL, Avesani CM. Aplicabilidade da avaliação global subjetiva e malnutrition inflammation score na avaliação do estado nutricional na doença renal crônica. J Bras Nefrol. 2014; 36(1):236-240.

27. Cristóvão AFAJ. Eficácia das restrições hídrica e dietética em pacientes renais crônicos em hemodiálise. Rev Bras Enferm. 2015; 68(6):1154-1162. 
28. Freitas ATVS, VAZ IMF, Ferraz FS, Peixoto MRG, Campos MIVM. Prevalence of malnutrition and associated factors in hemodialysis patients. Rev Nutr. 2014; 27(3): 357 -366.

29. Raff AC, Lieu S, Melamed ML. et al. Relationship of impaired olfactory function in ESRD to malnutrition and retained uremic molecules. A J Kidney Dis. 2008; 52(1):102-110.

30. Burge JC, Park HS, Whitlock CP, Schemmel RA. Taste acuity in patients undergoing long-term hemodialysis. Kidney Int, 1979;15(1):49-53.

\section{CORRESPONDÊNCIA}

Mônica Batista Soares

Rua SV-2 Quadra 20, Lote 23,

Residencial Sonho Verde, Goiânia - Goiás. CEP: $74730-545$

E-mail: monica.nutri@hotmail.com 